\title{
Surface acoustic wave mediated coupling of free-space radiation into surface plasmon polaritons on plain metal films
}

\author{
C. Ruppert, ${ }^{1, *}$ J. Neumann, ${ }^{2}$ J. B. Kinzel, ${ }^{2}$ H. J. Krenner, ${ }^{2}$ A. Wixforth, ${ }^{2}$ and M. Betz ${ }^{1,3}$ \\ ${ }^{1}$ Physik-Department E11, Technische Universität München, James-Franck-Straße, D-85748 Garching, Germany \\ ${ }^{2}$ Lehrstuhl für Experimentalphysik 1, Universität Augsburg, Universitätsstr. 1, D-86159 Augsburg, Germany \\ ${ }^{3}$ Experimentelle Physik 2, Technische Universität Dortmund, Otto-Hahn-Str. 4, D-44227 Dortmund, Germany
}

(Received 2 July 2010; published 27 August 2010)

\begin{abstract}
We propose and demonstrate a surface acoustic wave-driven converter of light into surface plasmon polaritons. An otherwise unstructured thin metal film is deformed by traveling acoustic waves on a piezoelectric substrate underneath. This spatially periodic corrugation enables to bridge the momentum gap between freespace radiation and surface-bound modes. This principle is realized with plain gold films on a $\mathrm{LiNbO}_{3}$ wafer where surface acoustic waves induce surface ripples in the metal. For near-infrared light we observe efficiencies of order $10^{-4}$ for exciting surface plasmon polaritons.
\end{abstract}

DOI: 10.1103/PhysRevB.82.081416

PACS number(s): 73.20.Mf, 77.65.Dq

Collective electronic excitations propagating along the interface between a metal and a dielectric play a key role in various scientific areas. In particular, the controlled coupling of free-space radiation into surface plasmon polaritons (SPPs) is integral to developments in nanoplasmonics. Gratings represent a common and straightforward tool to overcome the wave-vector mismatch between light and SPPs and to, thereby, achieve efficient and broadband coupling into surface-bound modes. Active control of such couplers is intrinsically difficult because their performance is intimately linked to the geometry of the grating and the incident radiation. Very recently, all-optical techniques have been demonstrated to achieve coupling of free-space radiation to SPPs of unstructured, plain gold films. ${ }^{1,2}$ Those schemes rely on the effective momentum transfer between optical beams typical of four-wave mixing experiments, i.e., on transient lightinduced gratings. While this approach offers the unique advantage of picosecond launch windows for SPP excitations, its efficiency is limited by the weak dielectric contrast of optically induced gratings. We note that also acoustically induced plasmonic functionalities have been demonstrated before in a Kretschmann configuration. ${ }^{3}$ However, in contrast to the present study, this approach does not enable the conversion of free-space radiation into SPPs. We also note that the interaction of SPPs and coherent and incoherent phonons has been theoretically studied. ${ }^{4,5}$

In this Rapid Communication, we demonstrate coupling of free-space radiation into SPPs utilizing a dynamic grating induced by surface acoustic waves (SAWs). Specifically, SAWs are generated on a $\mathrm{LiNbO}_{3}$ wafer at a frequency of $f \approx 480 \mathrm{MHz}$ and induce a periodic deformation of a gold thin film on top. These surface ripples have an amplitude of about a nanometer and allow for the conversion of nearinfrared light into SPPs with $\sim 10^{-4}$ efficiency. Coupling is verified by the analysis of the $\mathrm{SAW}$-induced reflectivity changes for TM incident radiation. Spectral tuning of the coupler is achieved by changing the angle of incidence of the free-space radiation and/or modifying the radio frequency (RF) of the driving SAWs.

The dispersion of SPPs at a metal-dielectric interface with dielectric functions $\varepsilon_{\mathrm{m}}$ and $\varepsilon_{\mathrm{d}}$ is given by

$$
k_{\mathrm{SPP}}=k_{0} \sqrt{\frac{\varepsilon_{\mathrm{m}} \varepsilon_{\mathrm{d}}}{\varepsilon_{\mathrm{m}}+\varepsilon_{\mathrm{d}}}} .
$$

In the case of air $\varepsilon_{\mathrm{d}}$ is $\approx 1$. As a result, $k_{\mathrm{SPP}}$ is always larger than the wave vector $k_{0}=\omega / c$ of light and direct excitation of SPPs by free-space radiation is inhibited. A grating with period $\Lambda_{\mathrm{G}}$ provides an additional momentum $K_{\mathrm{G}}=2 \pi / \Lambda_{\mathrm{G}}$ to overcome this mode mismatch. Fulfilling the condition

$$
k_{\mathrm{SPP}}=k_{0} \sin (\theta) \pm m K_{\mathrm{G}}
$$

with an integer $m$, light with an off-normal angle of incidence $\theta$ couples resonantly to the SPP. This commonly applied method offers high conversion efficiencies and tunability of the resonance via the angle of incidence.

The guiding idea of the present study is to create a grating by electrically inducing SAWs on a piezoelectric solid [cf. Fig. 1(a)]. In particular, interdigital transducers (IDTs) on a piezoelectric substrate allow for the application of electric potentials alternating periodically in space and time. Matching the RF frequency to acoustic waves with a wavelength close to the IDT spacing leads to resonant excitation of SAWs. Several types of waves exist depending on the choice of the material and its crystallographic orientation of which the Rayleigh-type SAW is of particular importance in our case. For this SAW the particle displacement is elliptical along the direction of propagation and hence, the wave consists of a shear deformation normal to the surface and a density modulation. A thin gold film deposited on top of the crystal is periodically deformed by this SAW. Thus a propagating grating is defined with an adjustable amplitude up to a few nanometers and a period ranging from several tens of microns to a few hundreds of nanometers which, furthermore, provides the potential for nanosecond electrical switching.

Since the coupling efficiency for SPP generation increases with larger grating amplitudes, we use $\mathrm{LiNbO}_{3}$ in $128^{\circ} \mathrm{YX}$ cut which offers a very large electromechanical coupling coefficient of $K^{2}=5.6 \%$. The only SAW mode excited in this configuration is of Rayleigh type. ${ }^{6}$ For the SAW-induced grating constant and the optical wavelengths used in the ex- 

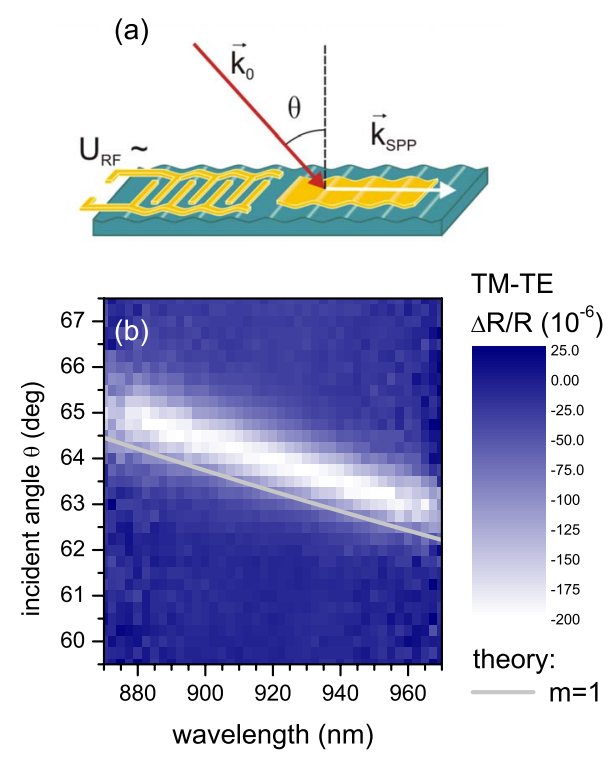

FIG. 1. (Color online) (a) Scheme of a SAW driven plasmon launcher: an RF voltage is applied to an interdigital transducer and generates surface acoustic waves that deform a metal film on top. This grating enables to convert free-space radiation $\left(\vec{k}_{0}\right)$ into propagating SPP modes $\left(\vec{k}_{\mathrm{SPP}}\right)$. (b) Difference of the normalized TM and TE reflectivity changes related to the SAW-induced grating. Solid line: theoretical prediction for the angular/spectral dependence of the resonance according to Eq. (2) and $m=1$.

periments, several constraints have to be considered. SAWs of larger period and correspondingly lower frequency lead to larger surface ripples favorable for SPP excitation. At the same time larger grating periods demand for larger offnormal angles of incidence and/or larger optical wavelengths [cf. Eq. (2)]. In this study, a grating period of $\sim 8 \mu \mathrm{m}$ in combination with near-infrared radiation from a Ti:sapphire laser turned out to be a good compromise. In particular, our device contains gold IDTs with 50 finger pairs and $4 \mu \mathrm{m}$ electrode separation to excite SAWs with a wavelength of $8 \mu \mathrm{m}$. Each electrode is $800-\mathrm{nm}$-wide and $250-\mu \mathrm{m}$-long. $1.7 \mathrm{~mm}$ from the IDT, a $300 \times 250 \mu \mathrm{m}^{2}$ and 40 -nm-thick gold film (on $10 \mathrm{~nm} \mathrm{Ti}$ adhesion layer) is deposited directly on the substrate.

A home-built femtosecond Ti:sapphire laser is used as a broadband light source covering a spectral range from 870 to $970 \mathrm{~nm} .{ }^{7}$ Both the sample mounting and the fiber-based detection optics are implemented on a $\theta-2 \theta$ stage to facilitate angular scans. Polarization optics controls the linear polarization state of the laser-pulse train with respect to the plane of incidence (TM and TE polarization). The sample is oriented such that the propagation direction of the SAWs coincides with the plane of incidence. The light is weakly focused on the sample with a spot size slightly smaller than the dimensions of the gold film, giving rise to an angular resolution of $0.7^{\circ}$ Spectral selection is provided either by bandpass filters with full width half maximum (FWHM) $10 \mathrm{~nm}$ width or by dispersing the reflected light in a grating spectrometer with $3.5 \mathrm{~nm}$ resolution. We use lock-in-detection referenced to a $2 \mathrm{kHz}$ amplitude modulation of the SAWs driven by a high-power RF generator. Essentially, this scheme extracts the SAW-induced reflectivity change in the gold film.

A two-dimensional representation of the reflectivity changes as a function of the angle of incidence and optical wavelength is presented in Fig. 1(b). For this set of data, the entire laser spectrum is used and the reflected light is analyzed in the spectrometer. The RF frequency is $f=490.276 \mathrm{MHz}$ and a power of $P=13 \mathrm{~mW}$ is applied to the device. While the result for TE polarized light is rather unstructured, the difference of the signals for TM and TE polarizations displayed in Fig. 1(b) shows a pronounced negative peak with an angular position that changes with wavelength. As will be corroborated by further results below, this dip points to the coupling of free-space radiation to SPP modes mediated by the SAW-induced grating. We note that the negative $\sim 10^{-4}$ signals directly prove missing reflected light for TM polarization as expected for SPP excitation. The observed spectral linewidth of the SPP resonance is $\Delta \lambda \approx 60 \mathrm{~nm}$. However, such a broad linewidth is not unexpected since we use a gold thin film. Model calculations for $40 \mathrm{~nm}$ gold on a dielectric substrate indicate the width of the SPP resonance to increase by a factor of three compared to bulk gold. In addition, SPP attenuation is very sensitive to surface roughness which in part is related the roughness of the optically polished substrate.

Figure 1(b) also shows the expected dispersion of the resonance angle according to Eq. (2) and $m=1$ which agrees reasonably well with the experimental trend. The dielectric function for gold used for the calculation of $k_{\mathrm{SPP}}$ in Eq. (1) is derived from a Drude fit to experimental data $^{8}$ while the grating periods in Eq. (2) are determined from diffraction experiments detailed below. At first glance, the grating period is given by the wavelength of the driving SAW according to $\Lambda_{\mathrm{G}}=v_{\mathrm{S}} / f_{\mathrm{SAW}}$, which, using $v_{\mathrm{S}}=3977 \mathrm{~m} / \mathrm{s},{ }^{9}$ yields $\Lambda_{\mathrm{G}}=8.112 \mu \mathrm{m}$. In the experiment, we directly measure the grating period on the gold film by analyzing the angular position of the first-order diffracted beam. For the given parameters we find $\Lambda_{\mathrm{G}}=7.57 \mu \mathrm{m}$, i.e., considerably smaller than expected. This phenomenon is caused by the metallized surface where the sound velocity is reduced due to electric short circuiting and mass loading. ${ }^{10}$ The velocity change $\Delta v$ is directly related to the electromechanical coupling coefficient via $K^{2}=2 \Delta v / v_{\mathrm{S}}$. The corresponding sound velocity of $3711 \mathrm{~m} / \mathrm{s}$ is in good agreement with previous results. ${ }^{11-13}$ The remaining angular discrepancy in Fig. 1(b) probably results from a resonance shift induced by the finite film thickness and surface roughness. ${ }^{14}$

A more detailed picture of the angular dependence of the SAW-induced reflectivity changes for both TE and TM polarization is displayed in Fig. 2(a). For this set of experiments, the incident light is filtered with a bandpass at $940 \mathrm{~nm}$ (FWHM $10 \mathrm{~nm}$ ). A RF signal with a frequency of $f=491.032 \mathrm{MHz}$ and a power of $P=20 \mathrm{~mW}$ is applied to the launching IDT. The TE results vary only modestly with the angle of incidence. In marked contrast, we observe prominent dips in the TM reflectivity changes at angles of $\theta=63.7^{\circ}$ and $\theta=49.8^{\circ}$. While the former resonance is consistent with the results shown in Fig. 1(b), the latter resonance is also indicative of SPP launching via a more complex mechanism. In particular, the resonance position is close 


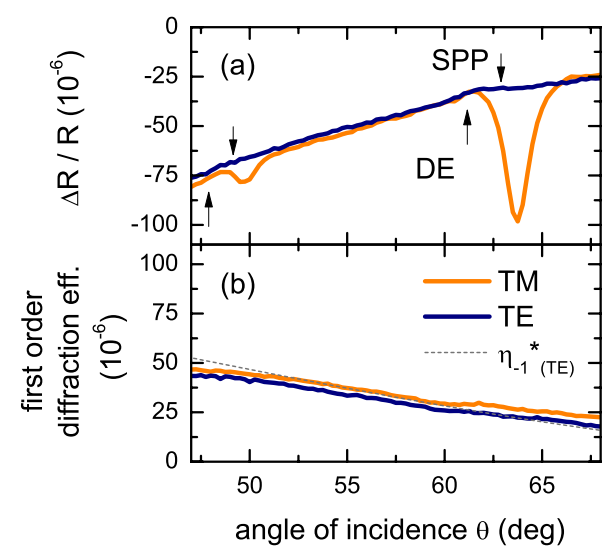

FIG. 2. (Color online) (a) Angular dependence of the SAWinduced normalized reflectivity change for TM- and TE-polarized light. The arrows pointing downwards (upwards) indicate the expected angular positions for the SPP (DE) resonances. (b) Angular dependence of the first-order diffraction efficiency $\eta_{-1}^{*}$. Dashed line: theoretical prediction for light of $\lambda=940 \mathrm{~nm}$ wavelength.

to the one expected for an $m=2$ coupling in Eq. (2). Such a direct process is unlikely to occur for a deformation induced by a mostly sinusoidal SAW. However, higher acoustic harmonics are easily generated on $\mathrm{LiNbO}_{3}$ in a nonlinear elastic process since the SAW power is confined to a layer of only a few acoustic wavelengths at the surface, resulting in quite large power densities close to the surface. The existence of second-harmonic SAWs is corroborated by the analysis of the diffraction pattern of the gold film. While no secondorder diffraction of the grating with the $\Lambda_{\mathrm{G}}=7.57 \mu \mathrm{m}$ period is resolved in the experiment, we clearly identify diffracted light consistent with the first-order diffraction of a $\Lambda_{\mathrm{G}}^{\prime}$ $=3.635 \mu \mathrm{m}$ grating. Together with the expected sound velocity dispersion of a mass-loaded film this periodicity is reasonable for the second harmonic of the driving SAW. Solving Eq. (2) with the measured grating periods of $\Lambda_{\mathrm{G}}$ $=7.57 \mu \mathrm{m}$ and $\Lambda_{\mathrm{G}}^{\prime}=3.635 \mu \mathrm{m}$, and $m=1$ predicts resonance angles of $\theta=62.9^{\circ}$ and $\theta=49.1^{\circ}$, respectively. As visualized by the arrows pointing downwards in Fig. 2(a), those angular positions agree well with the experiment.

The diffraction pattern of the SAW-induced grating reveals interesting details about the surface ripple on the metal film. In particular, the efficiency of diffraction into the first order provides information on the magnitude of the deformation. To this end, diffraction of light by SAWs is described by a phase grating with period $\Lambda_{\mathrm{G}}$ related to the surface ripple. ${ }^{15,16}$ For a sinusoidal grating with amplitude $\delta$ and reflection coefficient $R$ the first-order diffraction efficiency is

$$
\eta_{ \pm 1}=\frac{I_{ \pm 1}}{I_{0}} \approx R^{2}\left[k_{0} \cos (\theta) \cdot \delta\right]^{2} .
$$

Hence, a diffraction efficiency of $\eta_{-1}^{*}=I_{-1} / R I_{0}=2.2 \times 10^{-5}$ at $\theta=64^{\circ}$ incident angle for TM polarized light $(R=0.96)$ leads to an estimated amplitude of $\delta \approx 1.6 \mathrm{~nm}$. The theoretical angular dependence of the diffraction efficiency $\eta_{-1}^{*}$ for $\delta$ $=1.6 \mathrm{~nm}$ is shown in Fig. 2(b) and agrees well with the experimental results.

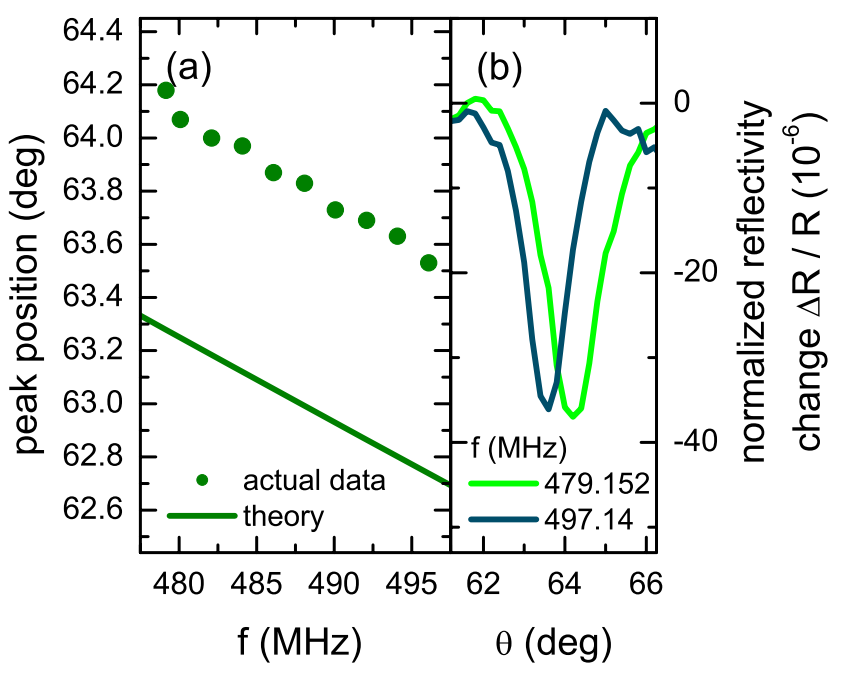

FIG. 3. (Color online) (a) Resonance angle for various frequencies and (b) exemplary angular scans for two different RF frequencies. Light is filtered via a bandpass at a central wavelength of $\lambda$ $=940 \mathrm{~nm}$ and $P=20 \mathrm{~mW}$ RF power is applied.

We note that apart from SPP excitation also diffraction edge (DE) anomalies may occur, i.e., anomalies for incident angles where light is diffracted right into the plane of the gold film. This is equivalent to the condition $k_{0}$ $=k_{0} \sin (\theta) \mp n K_{\mathrm{G}}$ with an integer $n$. The expected angles for the $\mathrm{DE}$ anomaly and the two above grating periods are $\theta$ $=61.1^{\circ}$ and $\theta=47.9^{\circ}$ [cf. arrows pointing upward in Fig. 2(b)]. Neither TM nor TE polarization show a clear signature of DE anomalies. This finding is not unexpected since DE anomalies for most experimental configurations are weaker than resonances related to SPP excitation. ${ }^{17}$

Now we turn to the tuning potential of the SAW-induced grating. Not only the amplitude of the grating can be controlled by varying the RF power but also the frequency and thus the grating period is slightly tunable. The corresponding SPP resonance angles for a variation in the frequency within the $\Delta f=18 \mathrm{MHz}$ bandwidth of the IDT are shown as symbols in Fig. 3(a). To obtain those resonance positions, angular scans for TM-and TE-polarized light at $\lambda=940 \mathrm{~nm}$ are performed and the peaks' angular positions are extracted after subtracting both curves. Examples for the SAW-induced reflectivity changes for TM polarized light are shown in Fig. 3(b). Most importantly, we observe an angular shift of the resonance position of up to $\theta=0.7^{\circ}$ upon tuning the RF frequency. We note that frequency tuning over the range of $f$ $=489 \mathrm{MHz} \pm 9 \mathrm{MHz}$ comes along with a $4 \%$ variation in $\Lambda_{\mathrm{G}}$. Within this interval, Eq. (2) predicts a linear shift of $\theta$ [solid line in Fig. 3(a)] in good agreement with the experimental trend. For constant RF power, a maximum peak amplitude is observed at $f \approx 489 \mathrm{MHz}$ which decreases for both higher and lower frequencies as the excitation of SAWs becomes less efficient. The overall observed shift of the SPP resonance is approximately half its angular peak width [cf. Fig. 3(b)] when $f$ is tuned across the IDTs bandwidth.

We also investigate the power dependence of the SPP resonance and the grating's diffraction efficiency for TM polarized light at $\lambda=490 \mathrm{~nm}$ wavelength (data not shown). 
First of all, we find the IDTs resonance frequency to shift by $\Delta f \approx 0.5 \mathrm{MHz}$ while the RF power is increased from $P$ $=0.1 \mathrm{~mW}$ to $P=20 \mathrm{~mW}$. Using a temperature coefficient of $72 \mathrm{ppm} / \mathrm{K}$ (Ref. 18) we estimate a temperature increase in $\Delta T=15 \mathrm{~K}$ for the largest $\mathrm{RF}$ power. For each excitation power and after appropriate adjustment of the RF-driving frequency, we perform an angular scan to yield curves as shown in Figs. 2(a) and 3(b) and in turn determine the corresponding strength of the resonance. Most importantly, both the first-order diffraction efficiency and the amplitude of the SPP resonance increase linearly with the applied RF power. For the case of diffraction, this trend is understood from Eq. (3) where $\eta_{-1} \propto \delta^{2}$. According to existing literature ${ }^{19,20}$ the SAW power is related to $\delta$ as $P_{\mathrm{SAW}}=C f_{\mathrm{SAW}} \delta^{2}$ with $C$ being a material-dependent constant, hence the diffraction efficiency is indeed expected to increase linearly with RF power. Remarkably, the amplitude of the SPP resonance follows the same behavior. This result coincides with a theoretical prediction for small grating amplitudes. ${ }^{21}$

In conclusion, we have demonstrated an electrically switchable SPP launcher based on traveling SAWs that induce spatially periodic deformations on an otherwise plain metal film. The conversion efficiency between near-infrared light and surface-bound modes is $2 \times 10^{-4}$ and is only limited by the RF power delivered to the device. Our results offer different insight into the potential use of SAWs in the emerging field of active plasmonics.

We acknowledge stimulating discussions with N. Rotenberg. This work has been supported by the DFG within the priority program 1391 "Ultrafast Nanooptics", by the Mercur Research Center Ruhr (MERCUR) under the Contract No. An-2010-0028 and by the Nanostructure Initiative Munich (NIM).
*Corresponding author; claudia.ruppert@mytum.de

${ }^{1}$ J. Renger, R. Quidant, N. van Hulst, S. Palomba, and L. Novotny, Phys. Rev. Lett. 103, 266802 (2009).

${ }^{2}$ N. Rotenberg, M. Betz, and H. M. van Driel, Phys. Rev. Lett. 105, 017402 (2010).

${ }^{3}$ X. Sun, S. Shiokawa, and Y. Matsui, J. Appl. Phys. 69, 362 (1991).

${ }^{4}$ A. V. Chaplik and M. V. Krasheninnikov, Surf. Sci. 98, 533 (1980).

${ }^{5}$ A. M. Marvin, F. Nizzoli, and L. Giovannini, Phys. Rev. B 51, 10134 (1995).

${ }^{6} \mathrm{~K}$. Hashimoto, Surface Acoustic Wave Devices in Telecommunications: Modelling and Simulation (Springer, New York, 2000).

${ }^{7}$ C. Ruppert and M. Betz, Opt. Express 16, 5572 (2008).

${ }^{8}$ P. B. Johnson and R. W. Christy, Phys. Rev. B 6, 4370 (1972).

${ }^{9}$ J. Temmyo, I. Kotaka, T. Inamura, and S. Yoshikawa, IEEE Trans. Sonics Ultrason. 27, 218 (1980).

${ }^{10}$ J. J. Campbell and W. R. Jones, IEEE Trans. Sonics Ultrason. 15, 209 (1968).

${ }^{11}$ S. Kakio, H. Nakamura, Y. Kanamori, and Y. Nakagawa, Jpn. J. Appl. Phys., Part 1 38, 3261 (1999).
${ }^{12}$ K. Yamanouchi and K. Shibayama, J. Appl. Phys. 43, 856 (1972).

${ }^{13}$ A. J. Slobodnik, E. D. Conway, and R. T. Delmonico, Microwave Acoustics Handbook (Air Force Cambridge Research Laboratories, Bedford, MA 1973), Vol. 1A.

${ }^{14}$ A. Hoffmann, Z. Lenkefi, and Z. Szentirmay, J. Phys.: Condens. Matter 10, 5503 (1998).

${ }^{15}$ E. G. H. Lean, in Progress in Optics XI, edited by E. Wolf (North-Holland, Amsterdam, 1973), pp. 123-166.

${ }^{16}$ G. I. Stegeman, IEEE Trans. Sonics Ultrason. 23, 33 (1976).

${ }^{17}$ N. Rotenberg, J. N. Caspers, and H. M. van Driel, Phys. Rev. B 80, 245420 (2009).

${ }^{18}$ R. Veith, K. Wagner, and F. Seifert, in Piezoelectricity, Evolution and Future of a Technology, edited by W. Heywang, K. Lubitz, and W. Wersing (Springer, New York, 2008), pp. 311-350.

${ }^{19}$ R. M. White, IEEE Trans. Electron Devices 14, 181 (1967).

${ }^{20}$ E. G. H. Lean and C. G. Powell, Proc. IEEE 58, 1939 (1970).

${ }^{21}$ A. V. Krasavin, K. F. MacDonald, and N. I. Zheludev, in Nanophotonics with Surface Plasmons, edited by V. M. Shalaev and S. Kawata (Elsevier, New York, 2007), pp. 110-139. 